\title{
Validation of Disposable Stochastic Sensors Based on Nanolayer Depositon(s) of Silver and AgC Composite on Plastic for the Assay of $\alpha$-Amylase in Whole Blood and Saliva ${ }^{\dagger}$
}

\author{
Alexandrina Lungu-Moscalu ${ }^{1,2, *}$, Raluca-Ioana Stefan-van Staden ${ }^{1,2}$ and Marius Badulescu ${ }^{3}$ \\ 1 Faculty of Applied Chemistry and Material Science, University Politehnica of Bucharest, Bucharest 060021, \\ Romania; ralucavanstaden@gmail.com \\ 2 Laboratory of Electrochemistry and PATLAB, National Institute of Research for Electrochemistry and \\ Condensed Matter, 202, Splaiul Independentei Str., Bucharest 060021, Romania \\ 3 Low Temperature Plasma Laboratory, National Institute for Lasers, Plasma and Radiation Physics \\ (NILPRP), 409 Atomistilor St., Magurele 077125, Romania; bad_mariuss@yahoo.com \\ * Correspondence: alex_ly15@yahoo.com; Tel.: +40-770508382 \\ + Presented at the Virtual Eurachem Workshop 2020 - “Quality Assurance for Analytical Laboratories in the \\ University Curriculum", 14-15 July 2020; Available online: https://eurachem2020.ro/.
}

Published: 17 September 2020

\begin{abstract}
Nanolayer deposition(s) of Ag and AgC composite on a plastic substrate was used to design disposable stochastic sensors. The first sensor was based on an Ag nanofilm deposited on plastic; for the second sensor, an $\mathrm{AgC}$ composite was deposited on plastic, and, for the third sensor, on the plastic was deposited first a nanofilm of $\mathrm{Ag}$ and on top of it a nanofilm of the $\mathrm{AgC}$ composite. $\alpha$-Cyclodextrin was used to modify the active surface of the sensor.
\end{abstract}

Keywords: interleukins; stochastic sensors; method validation

Wide linear concentration ranges were obtained as follows: for the assay of $\alpha$-amylase in whole blood: $1.00 \times 10^{-7}-1.00 \times 10^{3} \mathrm{U} \mathrm{mL}^{-1}$, and for the assay of $\alpha$-amylase in saliva: $1.5 \times 10^{-15}-1.5 \times 10^{2} \mathrm{U}$ $\mathrm{mL}^{-1} \cdot \alpha$-Amylase was reliably determined from whole blood and saliva samples using the proposed disposable stochastic sensors.

Validation of the proposed sensors and screening method was done using real samples of whole blood and saliva.

Acknowledgments: The authors want to thank for the support provided by UEFISCDI, PNCDI III framework, PN-III-P4- ID-PCE-2016-0120. A.L.-M. wants to thank the Operational Programme Human Capital of the Ministry of European Funds through the Financial Agreement 51668/09.07.2019, SMIS code 124705 for the financial support.

Conflicts of Interest: The author declares no conflict of interest.

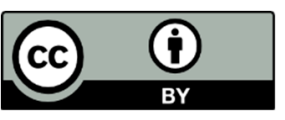

(C) 2020 by the authors. Licensee MDPI, Basel, Switzerland. This article is an open access article distributed under the terms and conditions of the Creative Commons Attribution (CC BY) license (http://creativecommons.org/licenses/by/4.0/). 